\title{
Phase II study of adjuvant chemotherapy of S-1 plus oxaliplatin for patients with stage III gastric cancer after D2 gastrectomy
}

\author{
Kohei Shitara ${ }^{1} \cdot K_{\text {Keisho }}$ Chin $^{2} \cdot$ Takaki Yoshikawa $^{3} \cdot$ Hitoshi Katai $^{4}$. \\ Masanori Terashima $^{5} \cdot$ Seiji Ito $^{6} \cdot$ Motohiro Hirao $^{7} \cdot$ Kazuhiro Yoshida $^{8}$. \\ Eiji Oki $^{9} \cdot$ Mitsuru Sasako ${ }^{10} \cdot$ Yasunori Emi ${ }^{11} \cdot$ Toshimasa Tsujinaka $^{12}$
}

Received: 17 September 2015/ Accepted: 17 November 2015/Published online: 1 December 2015

(C) The International Gastric Cancer Association and The Japanese Gastric Cancer Association 2015

\begin{abstract}
Background The Adjuvant Chemotherapy Trial of S-1 for Gastric Cancer (ACTS-GC) demonstrated a survival benefit by adjuvant S-1 monotherapy in patients who had undergone curative resection of stage II/III gastric cancer, but there is still a need to improve the efficacy of treatment of stage III disease. We investigated the tolerability and safety of S-1 and oxaliplatin as adjuvant chemotherapy for stage III gastric cancer.

Methods Japanese patients with stage III gastric cancer who had undergone D2 or more extensive lymphadenectomy were enrolled. In the first cycle, S-1 $\left(40-60 \mathrm{mg} / \mathrm{m}^{2}\right.$ twice daily) alone was given orally for 2 weeks of a 3 -week cycle. From the second cycle, S-1 was administered as in the first cycle and oxaliplatin $\left(100 \mathrm{mg} / \mathrm{m}^{2}\right)$ was infused intravenously on day 1 . Treatment was continued
\end{abstract}

Electronic supplementary material The online version of this article (doi:10.1007/s10120-015-0581-1) contains supplementary material, which is available to authorized users.

Kohei Shitara

kouheis0824@yahoo.co.jp; kshitara@east.ncc.go.jp

1 Department of Gastroenterology and Gastrointestinal Oncology, National Cancer Center Hospital East, 6-5-1

Kashiwanoha, Kashiwa, Chiba 277-8577, Japan

2 Department of Gastroenterology, Cancer Institute Hospital of JFCR, Tokyo, Japan

3 Department of Gastrointestinal Surgery, Kanagawa Cancer Center, Yokohama, Japan

4 Gastric Surgery Division, National Cancer Center Hospital, Tokyo, Japan

5 Division of Gastric Surgery, Shizuoka Cancer Center, Shizuoka, Japan for 8 cycles. The primary end point was the treatment completion rate for eight cycles.

Results Sixty-three patients were enrolled and 62 patients were included in analysis. The treatment completion rate was $74.2 \%$, which was higher than the expected completion rate of $72.0 \%$. The median relative dose intensities were $77.1 \%$ for S-1 and $72.6 \%$ for oxaliplatin, with 41.9 and $61.7 \%$ patients requiring dose reduction of S-1 and oxaliplatin, respectively. Neutropenia was the only grade 3 or higher adverse event with an incidence $10 \%$ or greater $(32.3 \%)$. There was no grade 3 or higher peripheral sensory neuropathy or treatment-related death.

Conclusions S-1 and oxaliplatin therapy is suggested to be manageable and safe with optimal dose reduction and delay in selected patients for stage III gastric cancer after D2 gastrectomy, and warrants further evaluation in larger studies.

Keywords Stage III gastric cancer - Adjuvant chemotherapy $\cdot$ Oxaliplatin $\cdot \mathrm{S}-1 \cdot \mathrm{S}-1$ and oxaliplatin

6 Department of Gastroenterological Surgery, Aichi Cancer Center Hospital, Nagoya, Japan

7 Department of Surgery, National Hospital Organization Osaka National Hospital, Osaka, Japan

8 Department of Surgical Oncology, Gifu University Graduate School of Medicine, Gifu, Japan

9 Department of Surgery and Science, Graduate School of Medical Sciences, Kyushu University, Fukuoka, Japan

10 Department of Surgery, Hyogo College of Medicine, Nishinomiya, Japan

11 Department of Surgery, Saiseikai Fukuoka General Hospital, Fukuoka, Japan

12 Department of Surgery, Kaizuka City Hospital, Osaka, Japan 


\section{Introduction}

Gastric cancer is a disease with high morbidity and mortality rates [1]. In Japan, gastric cancer is the third-leading cause of cancer death for both men and women [2]. Surgical resection is the first choice for localized gastric cancer in Japan, and adjuvant chemotherapy is recommended for stage II or stage III disease after curative resection [3].

S-1 is an oral anticancer agent that contains tegafur, a prodrug of 5-fluorouracil, combined with two modulators (gimeracil and oteracil) [4]. The Adjuvant Chemotherapy Trial of S-1 for Gastric Cancer (ACTS-GC) compared surgery plus adjuvant S-1 with surgery alone in patients who had undergone curative gastrectomy with D2 lymph node dissection for stage II or stage III gastric cancer. In that study, adjuvant $\mathrm{S}-1$ demonstrated a significant survival benefit over surgery alone [5, 6]. Therefore, adjuvant S-1 has become a standard treatment for patients undergoing curative resection of stage II or stage III gastric cancer [3]. However, the efficacy of S-1 is still limited, with a 5-year overall survival rate of $67.1 \%$ for patients with stage IIIA disease and $50.2 \%$ for patients with stage IIIB disease. Also, the treatment benefit of S-1 seems to be smaller in stage IIIA or stage IIIB disease than in stage II disease as suggested by ACTS-GC [3,6]. Thus, the treatment for patients with stage III disease needs further improvement [6].

Several studies have been conducted to explore combination chemotherapy as adjuvant chemotherapy for gastric cancer. The CLASSIC study compared surgery alone and surgery plus adjuvant therapy with capecitabine and oxaliplatin in patients who had undergone curative resection of stage II, IIIA, or IIIB gastric cancer with D2 lymph node dissection. The 3-year disease-free survival as the primary end point was significantly improved with capecitabine and oxaliplatin therapy [7]. In Japan, a feasibility study was conducted to investigate the tolerability of adjuvant therapy with S-1 and cisplatin (SP). In that study, because of to toxic effects observed mainly during the first cycle of SP therapy, the treatment completion rate was lower than expected. Therefore, the treatment schedule was revised during the study period to include S-1 monotherapy in the first cycle and subsequent SP therapy in the second to fourth cycles, which resulted in an increase of the treatment completion rate [8]. Meanwhile, a randomized phase III study suggested that S-1 and oxaliplatin (SOX) was almost as effective as SP, and with a more favorable safety profile, as the first-line treatment of advanced gastric cancer $(\mathrm{G}-$ SOX study) [9]. Compared with SP therapy, SOX therapy has several benefits, such as unnecessary hydration during platinum agent administration, low renal toxicity, and little limitation due to renal function for its use. Therefore, we conducted a phase II study to evaluate the tolerability and safety of SOX therapy as adjuvant therapy after curative resection of stage III gastric cancer.

\section{Methods}

\section{Study design and patients}

This study was a single-arm, prospective, multicenter phase II study at 11 centers in Japan. The objective was to evaluate the tolerability and safety of SOX therapy as an adjuvant therapy in stage III gastric cancer patients who had undergone curative resection.

The inclusion criteria were histologically confirmed stage III gastric cancer (excluding T4bN2/N3 disease), R0 surgery with D2 or more extensive lymphadenectomy, age of 20 years or older, and Eastern Cooperative Oncology Group performance status of $0-1$. Patients were also required to have adequate hematologic function (leukocyte count of $12,000 / \mathrm{mm}^{3}$ or lower, neutrophil count of $1,500 /$ $\mathrm{mm}^{3}$ or higher, platelet count of $100,000 / \mathrm{mm}^{3}$ or higher, and hemoglobin level of $9.0 \mathrm{~g} / \mathrm{dL}$ or higher), hepatic function [total bilirubin level of 1.5 times the institutional upper limit of normal (ULN) or lower, aspartate aminotransferase or alanine aminotransferase level of 3.0 times the ULN or lower, and alkaline phosphatase level of 2.5 times the ULN or lower], and renal function (serum creatinine level less than the ULN and creatinine clearance of $50 \mathrm{~mL} / \mathrm{min}$ or greater). Disease stage was determined according to the 14th edition of the Japanese Classification of Gastric Carcinoma [10], which was different from the classification used in ACTS-GC (13th edition), resulting in a slightly different stage III population between this study and the previous study. Patients were enrolled within 6 weeks after undergoing gastrectomy and administration started from 4 weeks to within 7 weeks after the operation. Patients were excluded if they had any of the following conditions: a history of any treatment other than surgery for gastric cancer; difficulty in taking orally administered S-1; or severe drug hypersensitivity or peripheral sensory neuropathy.

This study protocol was approved by the institutional review boards of all participating institutions, and the study was conducted in accordance with the Helsinki Declaration and the good clinical practice guidelines. All patients provided written informed consent before the start of the study. This study was registered with the Japan Pharmaceutical Information Center (JapicCTI-132122). 


\section{Treatment and assessment}

On the basis of the results of the previous SP feasibility study [8], the study treatment consisted of S-1 monotherapy in the first cycle and S-1 combined with oxaliplatin in the second and subsequent cycles. In the first cycle, S-1 was given orally twice daily for 2 weeks of a 3-week cycle. The dosage of S-1 was $80 \mathrm{mg} /$ day for patients with a body surface area (BSA) of less than $1.25 \mathrm{~m}^{2}, 100 \mathrm{mg} / \mathrm{day}$ for patients with a BSA of $1.25 \mathrm{~m}^{2}$ or more and but less than $1.5 \mathrm{~m}^{2}$, and $120 \mathrm{mg} /$ day for patients with a BSA of $1.5 \mathrm{~m}^{2}$ or more. In the second and subsequent cycles, S-1 was administered according to the same schedule as in the first cycle and oxaliplatin $\left(100 \mathrm{mg} / \mathrm{m}^{2}\right)$ was infused intravenously for $2 \mathrm{~h}$ on day 1 . Before infusion of oxaliplatin, antiemetics (e.g., a 5-hydroxytryptamine ${ }_{3}$ receptor antagonist and dexamethasone) were administered prophylactically to prevent nausea and vomiting. Treatment was continued for eight cycles unless any of the discontinuation criteria were met: the patient experienced a recurrence of the underlying cancer; the patient could not resume treatment within 3 weeks after the scheduled first day of the next cycle; the patient required more than a two-level dose reduction of S-1; grade 4 peripheral sensory neuropathy occurred; the patient required a prohibited therapy; the patient could not visit the medical institution regularly; the patient refused further treatment; or a decision by the study investigator. The dose reduction criteria for S-1 and oxaliplatin were similar to those used in the G-SOX study [9]. The doses of both drugs were reduced if the platelet count was less than $25,000 / \mathrm{mm}^{2}$, if the neutrophil count was less than $500 / \mathrm{mm}^{2}$, or if grade 3-4 febrile neutropenia, diarrhea, or stomatitis occurred. The dose of oxaliplatin was reduced if the platelet count was less than $75,000 / \mathrm{mm}^{2}$ on day 29 with delayed initiation of the next treatment cycle or if grade 2 peripheral sensory neuropathy was noted on the first day of the cycle. The dose was reduced by $25 \mathrm{mg} / \mathrm{m}^{2}$ per level for oxaliplatin. If a third dose reduction of oxaliplatin was needed, the treatment was continued without oxaliplatin. The dose of S-1 was reduced by one dose level (levels 1 and 2 were as follows: 50 and $40 \mathrm{mg} /$ day for patients with a BSA of less than $1.25 \mathrm{~m}^{2}, 80$ and $50 \mathrm{mg} /$ day for patients with a BSA of $1.25 \mathrm{~m}^{2}$ or more and but less than $1.5 \mathrm{~m}^{2}$, and 100 and $80 \mathrm{mg} /$ day for patients with a BSA of $1.5 \mathrm{~m}^{2}$ or more). Details of the dose modification schedule are given in Tables S1 and S2.

During the study period, hematology tests, biochemistry tests, and assessment of symptoms and signs were performed before initiation of each cycle. The presence or absence of disease recurrence was evaluated at 6 months after surgery on the basis of the results of computed tomography or magnetic resonance imaging. However, if a patient discontinued treatment within 6 months and underwent final evaluation at that time, imaging at 6 months after surgery was not required. Adverse events were evaluated according to the Common Toxicity Criteria for Adverse Events, version 4.03.

\section{Statistical analysis}

The primary end point was the treatment completion rate, which was defined as the percentage of patients who completed eight cycles of SOX therapy, and included patients who continued with S-1 monotherapy after discontinuation of oxaliplatin therapy. The point estimate and $95 \%$ confidence interval $(\mathrm{CI})$ were calculated. Secondary end points were the ratio of delivered dose to the planned dose and safety. The planned dose was defined as the total dose if eight cycles had been completed without dose reduction. As exploratory analysis, we also assessed the relative dose intensity, which was defined as the ratio of delivered dose intensity to the planned dose intensity. Dose intensity was calculated as the ratio of the cumulative dose to the treatment duration per 21 days. Subgroup analyses of tolerability and safety were performed. The subgroups were defined on the basis of the following baseline patient characteristics: sex, age (younger than 65 years vs 65 years or older, younger than 75 years vs 75 years or older), BSA (less than $1.25 \mathrm{~m}^{2}, 1.25 \mathrm{~m}^{2}$ or greater to $1.5 \mathrm{~m}^{2}, 1.5 \mathrm{~m}^{2}$ or greater), Eastern Cooperative Oncology Group performance status, tumor histologic features, disease stage, tumor category, node category, type of gastrectomy, weight change after surgery (loss of less than $15 \%$ vs loess of $15 \%$ or more), and creatinine clearance (less than $70 \mathrm{~mL} /$ min vs $70 \mathrm{~mL} / \mathrm{min}$ or more). In exploratory analysis, we also assessed the proportion of patients with dose reductions by these subgroups.

The target sample size was designed to have appropriate precision for the treatment completion rate estimate. In this study, the SOX regimen was considered as feasible if the point estimate of the treatment completion rate was higher than $72.0 \%$, which was based on the actual treatment completion rates in ACTS-GC (77.9\%) and the CLASSIC study $(67 \%)$. Assuming the expected treatment completion rate of $72.0 \%$ and the range of the accurate $95 \% \mathrm{CI}$ of the point estimate for the treatment completion rate of $25 \%$ or less, we calculated the required sample size to be 55 patients. The target sample size was set at 60 patients to allow for some dropouts.

Safety was assessed in all of the patients who received at least one dose of S-1 or oxaliplatin (safety analysis set). The treatment completion rate and the ratio of delivered dose to the planned dose were analyzed for the patients who met the main inclusion criteria within the safety analysis set (full analysis set). Statistical analysis was 
performed with SAS version 9.2 (SAS Institute, Cary, NC, USA).

\section{Results}

\section{Patients}

Between July 2013 and February 2014, 63 patients were enrolled from 11 centers in Japan. One patient withdrew informed consent before receiving treatment, and 62 patients were included in the safety analysis set and the full analysis set. The patient characteristics are shown in Table 1. The median age was 64.5 years (range 22-81 years). According to the Japanese Classification of Gastric Carcinoma (14th edition), 17 patients $(27.4 \%)$ had stage IIIA disease, 22 patients $(35.5 \%)$ had stage IIIB disease, and 23 patients $(37.1 \%)$ had stage IIIC disease. Total gastrectomy and distal gastrectomy were performed in 32 patients $(51.6 \%)$ and 30 patients $(48.4 \%)$, respectively.

\section{Tolerability}

Forty-six patients from the full analysis set completed eight cycles of treatment, and the treatment completion rate was $74.2 \%$ (95\% CI 61.5-84.5\%), which was higher than the expected completion rate of $72.0 \%$. In 16 patients who could not complete the treatment, the reasons for discontinuation were as follows: treatment could not be resumed more than 3 weeks after the scheduled first day of the next cycle in seven patients (because of neutropenia in five patients, nausea in one patient, and thoracic empyema in one patient), four patients withdrew informed consent, the investigators decided that treatment should be discontinued in three patients, two patients showed recurrence, and one patient required anticoagulation because of pulmonary embolism. Forty-four of the 46 patients from the full analysis set who completed S-1 therapy also completed eight cycles of SOX therapy without discontinuation of oxaliplatin therapy $(71.0 \%, 95 \%$ CI 58.1-81.8\%). In the other two patients, oxaliplatin therapy was discontinued because of the investigator's judgment because of adverse events. The mean and median ratios of delivered dose to the planned dose were 77.2 and $87.5 \%$, respectively, for S-1 and 71.2 and $78.6 \%$, respectively, for oxaliplatin. The mean and median relative dose intensities were 75.6 and $77.1 \%$, respectively, for $\mathrm{S}-1$ and 71.2 and $72.6 \%$, respectively, for oxaliplatin.

Twenty-six patients $(41.9 \%)$ required reduction of the S-1 dose and eight patients $(12.9 \%)$ had reduction by two dose levels. In all of these patients, dose reduction was due
Table 1 Patient characteristics

\begin{tabular}{|c|c|c|}
\hline Characteristic & Number & Percentage \\
\hline \multicolumn{3}{|l|}{ Sex } \\
\hline Male & 39 & 62.9 \\
\hline Female & 23 & 37.1 \\
\hline \multicolumn{3}{|c|}{ Body surface area $\left(\mathrm{m}^{2}\right)$} \\
\hline$<1.25$ & 2 & 3.2 \\
\hline$\geq 1.25$ to $<1.5$ & 18 & 29.0 \\
\hline$\geq 1.5$ & 42 & 67.7 \\
\hline \multicolumn{3}{|c|}{ ECOG performance status } \\
\hline 0 & 56 & 90.3 \\
\hline 1 & 6 & 9.7 \\
\hline \multicolumn{3}{|c|}{ Tumor histologic type } \\
\hline Intestinal type & 20 & 32.3 \\
\hline Diffuse type & 42 & 67.7 \\
\hline \multicolumn{3}{|l|}{ 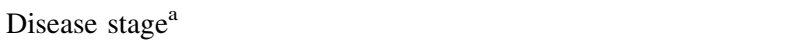 } \\
\hline IIIA & 17 & 27.4 \\
\hline IIIB & 22 & 35.5 \\
\hline IIIC & 23 & 37.1 \\
\hline \multicolumn{3}{|l|}{ Tumor category } \\
\hline $\mathrm{T} 2$ & 2 & 3.2 \\
\hline $\mathrm{T} 3$ & 21 & 33.9 \\
\hline $\mathrm{T} 4 \mathrm{a}$ & 39 & 62.9 \\
\hline \multicolumn{3}{|l|}{ Node category } \\
\hline N1 & 5 & 8.1 \\
\hline $\mathrm{N} 2$ & 21 & 33.9 \\
\hline N3a & 23 & 37.1 \\
\hline $\mathrm{N} 3 \mathrm{~b}$ & 13 & 21.0 \\
\hline \multicolumn{3}{|c|}{ Type of gastrectomy } \\
\hline Total & 32 & 51.6 \\
\hline Distal & 30 & 48.4 \\
\hline \multicolumn{3}{|c|}{ Rate of change in body weight after surgery (\%) } \\
\hline$<15$ & 60 & 96.8 \\
\hline$\geq 15$ & 1 & 1.6 \\
\hline Unknown & 1 & 1.6 \\
\hline \multicolumn{3}{|c|}{ Creatinine clearance $(\mathrm{mL} / \mathrm{min})$} \\
\hline $50-59$ & 11 & 17.7 \\
\hline $60-69$ & 8 & 12.9 \\
\hline$\geq 70$ & 43 & 69.4 \\
\hline
\end{tabular}

The median age was 64.5 years (range $22-81$ years)

ECOG Eastern Cooperative Oncology Group

a Japanese Classification of Gastric Carcinoma (14th edition)

to adverse events, mainly neutropenia and anorexia. Thirtyseven patients $(61.7 \%)$ required reduction of oxaliplatin dose and 16 patients $(26.7 \%)$ had reduction by two dose levels. The main reasons for dose reduction were neutropenia, peripheral sensory neuropathy, and anorexia. 
In five patients, dose reduction of S-1 was required at the start of the second cycle because of adverse events related to S-1 that had occurred during the first cycle. The adverse events that led to dose reduction were anorexia (two cases), malaise, nausea, elevation of C-reactive protein level, diarrhea, and neutropenia (one case each); the dose was reduced because of two or more adverse events in two patients.

Fifty-one patients $(82.3 \%)$ experienced a delay in the initiation of a subsequent cycle. The numbers of patients who experienced a delay after each cycle are as follows: 17 of 60 patients $(28.3 \%)$ for cycle 1,20 of 56 patients $(35.7 \%)$ after cycle 2,23 of 56 patients $(41.1 \%)$ after cycle 3,20 of 53 patients $(37.7 \%)$ after cycle 4,24 of 51 patients $(47.1 \%)$ after cycle 5,18 of 47 patients $(38.3 \%)$ after cycle 6 , and 26 of 46 patients $(56.5 \%)$ after cycle 7 . The commonest reasons for delays in administration were adverse events, mainly neutropenia.

\section{Safety}

Adverse events were reported by all 62 patients. Grade 3 adverse events occurred in 39 patients $(62.9 \%)$ with no grade 4 or higher events. Adverse events of all grades with an incidence of $10 \%$ or greater are shown in Table 2. Among gastrointestinal adverse events, grade 3 anorexia, nausea, and diarrhea were observed in four patients $(6.5 \%)$, three patients $(4.8 \%)$, and one patient $(1.6 \%)$, respectively. Neutropenia was the only grade 3 or higher hematologic toxicity with an incidence of $10 \%$ or more, and it occurred in 20 patients (32.3\%). Peripheral sensory neuropathy of all grades developed in 53 patients $(85.5 \%)$, but all events were of grade 2 or lower. Serious adverse events occurred in eight patients. All of the serious adverse events required hospitalization or prolongation of hospitalization. There were no treatment-related deaths. Two patients discontinued treatment because of recurrence (in the liver and in the liver plus lymph nodes, respectively).

\section{Subgroup analyses}

Results from exploratory subgroup analyses are shown in Table 3, and the full results are given in Table S3. The treatment completion rate was $71.0 \%$ for patients younger than 65 years and $77.4 \%$ for those 65 years or older, whereas the rate was $68.8 \%$ for patients who underwent total gastrectomy and $80.0 \%$ for those who underwent distal gastrectomy.

In the patients aged 65 years or older, dose reduction of S-1 and oxaliplatin was performed more frequently than in those younger than 65 years [54.8\% (17/31) vs $29.0 \%$ (9/ $31)$ for S-1, $71.0 \%(22 / 31)$ vs $48.4 \%(15 / 31)$ for
Table 2 Adverse events of all grades occurring in at least $10 \%$ of patients

\begin{tabular}{|c|c|c|c|c|}
\hline & \multicolumn{2}{|c|}{ All grades } & \multicolumn{2}{|c|}{ Grade 3} \\
\hline & $n$ & $\%$ & $n$ & $\%$ \\
\hline Leukopenia & 44 & 71.0 & 2 & 3.2 \\
\hline Neutropenia & 49 & 79.0 & 20 & 32.3 \\
\hline Anemia & 35 & 56.5 & 2 & 3.2 \\
\hline Thrombocytopenia & 29 & 46.8 & 3 & 4.8 \\
\hline Increased AST level & 34 & 54.8 & 1 & 1.6 \\
\hline Increased ALT level & 21 & 33.9 & 0 & 0.0 \\
\hline Increased ALP level & 22 & 35.5 & 1 & 1.6 \\
\hline Increased bilirubin level & 9 & 14.5 & 1 & 1.6 \\
\hline Decreased weight & 40 & 64.5 & 3 & 4.8 \\
\hline Diarrhea & 34 & 54.8 & 1 & 1.6 \\
\hline Stomatitis & 16 & 25.8 & 0 & 0.0 \\
\hline Nausea & 26 & 41.9 & 3 & 4.8 \\
\hline Vomiting & 9 & 14.5 & 0 & 0.0 \\
\hline Anorexia & 35 & 56.5 & 4 & 6.5 \\
\hline Malaise & 19 & 30.6 & 0 & 0.0 \\
\hline Infusion reaction & 29 & 46.8 & 0 & 0.0 \\
\hline Fatigue & 19 & 30.6 & 3 & 4.8 \\
\hline Hypokalemia & 18 & 29.0 & 3 & 4.8 \\
\hline Peripheral sensory neuropathy & 53 & 85.5 & 0 & 0.0 \\
\hline Dysgeusia & 22 & 35.5 & 0 & 0.0 \\
\hline Hypertension & 23 & 37.1 & 2 & 3.2 \\
\hline
\end{tabular}

$\overline{A L P}$ alkaline phosphatase, $A L T$ alanine aminotransferase, $A S T$ aspartate aminotransferase

oxaliplatin]. The percentage of patients who had dose reduction of S-1 and oxaliplatin after total gastrectomy was $43.8 \%(14 / 32)$ and $46.9 \%$ (15/32), respectively, whereas it was $40.0 \%(12 / 30)$ and $73.3 \%(22 / 30)$, respectively, after distal gastrectomy.

\section{Discussion}

To the best of our knowledge, this is the first study to evaluate the tolerability and safety of SOX therapy as adjuvant chemotherapy for Japanese patients with stage III gastric cancer. The treatment completion rate was $74.2 \%$ (95\% CI 61.5-84.5\%), which exceeded the expected treatment completion rate of $72.0 \%$. The frequency and severity of the adverse events observed in this study were similar to the known safety profile of SOX therapy in patients with advanced gastric cancer $[9,11]$. Neutropenia was the only grade 3 or higher adverse event with an incidence $10 \%$ or more, and the incidence $(32.3 \%)$ did not differ greatly from the incidence reported in previous studies of adjuvant fluoropyrimidine plus platinum agents after curative resection of gastric cancer $(22 \%$ reported by 
Table 3 Tolerability by age and type of gastrectomy

\begin{tabular}{|c|c|c|c|c|c|c|}
\hline & \multirow[t]{3}{*}{$n$} & \multirow[t]{3}{*}{ Treatment completion rate $(\%)$} & \multicolumn{4}{|c|}{ Ratio of delivered dose to the planned dose $(\%)$} \\
\hline & & & \multicolumn{2}{|c|}{ Mean } & \multicolumn{2}{|c|}{ Median } \\
\hline & & & S-1 & Oxaliplatin & S-1 & Oxaliplatin \\
\hline \multicolumn{7}{|c|}{ Age (years) } \\
\hline$<65$ & 31 & 71.0 & 77.3 & 70.8 & 88.8 & 75.0 \\
\hline$\geq 65$ & 31 & 77.4 & 77.1 & 71.7 & 87.5 & 78.6 \\
\hline \multicolumn{7}{|c|}{ Type of gastrectomy } \\
\hline Total & 32 & 68.8 & 72.4 & 67.4 & 87.3 & 73.6 \\
\hline Distal & 30 & 80.0 & 82.3 & 75.3 & 88.6 & 78.6 \\
\hline
\end{tabular}

Bang et al. [7] and $37 \%$ reported by Takahari et al. [8]). Peripheral sensory neuropathy, an adverse event specific to oxaliplatin, did not occur at a severity of grade 3 or higher, and both the incidence and the severity of myelosuppression and other adverse events (excluding neutropenia) did not have a marked influence on the continuation of treatment. Thus, adverse events caused by adjuvant SOX therapy after curative resection of gastric cancer could be easily manageable throughout the entire study by dose modification according to the dose reduction criteria that have already been used for advanced gastric cancer.

With regard to the toxicity of S-1 soon after surgery for gastric cancer, with reference to the SP feasibility study [8], the treatment was commenced with S-1 monotherapy in the first cycle, whereas SOX therapy was given in the second and subsequent cycles. In all five patients who had S-1 dose reduction after the first cycle, the dose reductions were due to adverse events related to $\mathrm{S}-1$, including gastrointestinal toxicity in four patients. On the basis of the dose reductions of S-1 after the first cycle, the grade of the adverse events did not increase during the subsequent cycles for these four patients, which may contribute to the favorable treatment completion rate. Dose reduction was performed more frequently in patients aged 65 years or older, but the treatment completion rate was not lower than that in patients younger than 65 years. Appropriate dose reduction is important for completion of adjuvant SOX therapy.

Although the treatment completion rate was higher than expected, 26 of 62 patients $(41.9 \%)$ and 37 of 60 patients $(61.7 \%)$ required dose reduction of S-1 and oxaliplatin, respectively. Although about half of the patients required dose reduction, the median relative dose intensities were $77.1 \%$ for S-1 and $72.6 \%$ for oxaliplatin in this study. Additionally, 51 of 62 patients $(82.3 \%)$ required chemotherapy administration to be delayed mainly because of adverse events. Thus, optimal management including dose modification and delay is very important for patients who have undergone curative surgery for stage III gastric cancer. In this study, we did not investigate the efficacy, and we do not know whether adjuvant SOX therapy could improve the prognosis of gastric cancer patients compared with S-1 monotherapy, which is the current standard adjuvant treatment in Japan. A randomized controlled trial is required to evaluate the efficacy of adjuvant SOX therapy.

In conclusion, this study suggests that adjuvant SOX therapy is manageable and safe with optimal dose reduction and delay in selected patients with curative resection of stage III gastric cancer. Further investigations are warranted to evaluate the efficacy of SOX as adjuvant chemotherapy in larger studies.

Acknowledgments This study was supported by Yakult Honsha. We thank all the patients, clinicians, and support staff who participated in this study. We are also grateful to Yu Sakata, Ichinosuke Hyodo, and Fumitaka Nagamura for their helpful advice.

\section{Compliance with ethical standards}

Conflict of interest Takaki Yoshikawa has received grants from Yakult Honsha, Chugai Pharmaceutical, Ono Pharmaceutical, MSD, Eli Lilly, Novartis Pharma, Covidien, Daiichi Sankyos and Nihon Kayaku and personal fees from Yakult Honsha, Taiho Pharmaceutical, Chugai Pharmaceutical, Eli Lilly, Abbott, Takeda Pharmaceutical, Covidien, Johnson and Johnson, Kaken Pharmaceutical, and Secom Medical Systems. Seiji Ito has received a grant from Yakult Honsha. Kazuhiro Yoshida has received grants from Yakult Honsha, Taiho Pharmaceutical, Pfizer, Chugai Pharmaceutical, Bristol-Myers Squibb, Kyowa Hakko Kirin, Ono Pharmaceutical, Daiichi Sankyo, and Sanofi and personal fees from Yakult Honsha, Taiho Pharmaceutical, Pfizer, and Chugai Pharmaceutical, and has a consultant or advisory relationship with Taiho Pharmaceutical and La Roche. Eiji Oki has received lecturer fees from Taiho Pharmaceutical, Takeda Pharmaceutical, Merck Serono, Chugai Pharmaceutical, and Yakult Honsha. Mitsuru Sasako has received grants and personal fees from Yakult Honsha and Taiho Pharmaceutical. Toshimasa Tsujinaka has received consulting fees from Yakult Honsha and lecture fees from Taiho Pharmaceutical. All remaining authors declare that they have no conflict of interest.

Human rights statement and informed consent All procedures followed were in accordance with the ethical standards of the responsible committee on human experimentation (institutional and national) and with the Helsinki Declaration of 1964 and later versions. Informed consent or substitute for it was obtained from all patients for their being included in the study. 


\section{References}

1. Ferlay J, Soerjomataram I, Ervik M, Dikshit R, Eser S, Mathers C, et al. GLOBOCAN 2012 v1.0. Cancer Incidence Mortality Worldwide. IARC CancerBase. 2012. http://globocan.iarc.fr/ default.aspx. Accessed 4 April 2015.

2. Ministry of Health, Labour and Welfare. Summary of vital statistics 2013. http://www.mhlw.go.jp/toukei/saikin/hw/jinkou/ kakutei13/dl/11_h7.pdf. Accessed 4 Apr 2015.

3. Japanese Gastric Cancer Association. Japanese gastric cancer treatment guidelines (ver. 4). Tokyo: Kanehara publication. 2014.

4. Shirasaka T. Development history and concept of an oral anticancer agent S-1 (TS-1): its clinical usefulness and future vistas. Jpn J Clin Oncol. 2009;39:2-15.

5. Sakuramoto S, Sasako M, Yamaguchi T, Kinoshita T, Fujii M, Nashimoto A, et al. Adjuvant chemotherapy for gastric cancer with S-1, an oral fluoropyrimidine. $\mathrm{N}$ Engl $\mathrm{J}$ Med. 2007;357:1810-20.

6. Sasako M, Sakuramoto S, Katai H, Kinoshita T, Furukawa H, Yamaguchi T, et al. Five-year outcomes of a randomized phase III trial comparing adjuvant chemotherapy with S-1 versus surgery alone in stage II or III gastric cancer. J Clin Oncol. 2011;29:4387-93.

7. Bang YJ, Kim YW, Yang HK, Chung HC, Park YK, Lee KH, et al. Adjuvant capecitabine and oxaliplatin for gastric cancer after D2 gastrectomy (CLASSIC): a phase 3 open-label, randomised controlled trial. Lancet. 2012;379:315-21.

8. Takahari D, Hamaguchi T, Yoshimura K, Katai H, Ito S, Fuse N, et al. Feasibility study of adjuvant chemotherapy with S-1 plus cisplatin for gastric cancer. Cancer Chemother Pharmacol. 2011;67:1423-8.

9. Yamada Y, Higuchi K, Nishikawa K, Gotoh M, Fuse N, Sugimoto N, et al. Phase III study comparing oxaliplatin plus S-1 with cisplatin plus $\mathrm{S}-1$ in chemotherapy-naïve patients with advanced gastric cancer. Ann Oncol. 2015;26:141-8.

10. Japanese Gastric Cancer Association. Japanese classification of gastric carcinoma (14th edition). Tokyo: Kanehara publication. 2010.

11. Koizumi W, Takiuchi H, Yamada Y, Boku N, Fuse N, Muro K, et al. Phase II study of oxaliplatin plus S-1 as first-line treatment for advanced gastric cancer (G-SOX study). Ann Oncol. 2010;21:1001-5. 